\title{
Short-Term Bus Load Matching and Forecasting Model
}

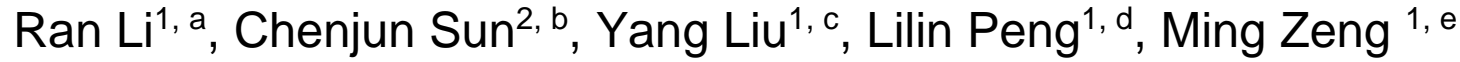 \\ ${ }^{1}$ School of Economics and Management, North China Electric Power University, Beijing 102206, \\ China; \\ ${ }^{2}$ State Grid Hebei Electric Power Company, Shijiazhuang 050000, Hebei Province, China.

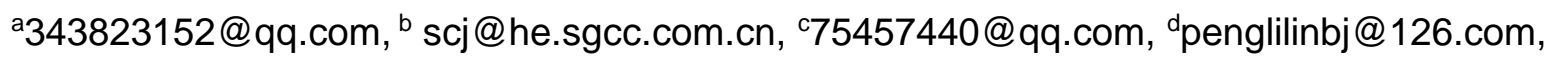 \\ e921735063@qq.com
}

\begin{abstract}
Bus load forecasting is the foundation to make power grid to be secure, economic and efficient, and also is the concrete measure and effective way to promote national energy saving and emissions reduction. Based on daily load curve of typical areas, and by means of subtractive clustering, the regional bus load was classified and bus load matching model was constructed. Furthermore, bus load forecasting model with the least squares support vector machine model (LSSVM) was put forward. Finally, the validity of load matching and forecasting model was verified by numerical examples.
\end{abstract}

Keywords: Short-term bus load forecasting; Least squares support vector machine (LSSVM); Matching model; Subtractive clustering.

\section{Introduction}

With a new round of power system reform progressed constantly and the energy saving and emissions reduction measures released continuously, the requirements of whole society on the safety, energy saving, economy, efficient operation of power grid and the entire power industry are increasing [1,2]. It is urgent to improve the bus load forecasting methods and models scientifically, and promote the scheduling system to develop timely and reasonable power generation plan, and implement scientific and effective generation dispatch. Bus load is the sum of the terminal load in a relatively small area, whose stability is weaker, load changing is rapider and trend is less obvious compared with the system load. In addition, the relevant factors affecting the bus load changing is also more varied and complex, such as temperature, rainfall, economic development and so on [3]. Therefore, how to implement scientific and effective bus load forecasting is an important research direction nowadays.

Compared with the system load forecasting, the bus load forecasting studies are relatively less. The traditional method is based on the system load forecasting to calculate the bus load according to the allocation coefficient. In recent years, such as expert systems, artificial neural networks, genetic algorithms, gray system and the others artificial intelligence algorithm are applied to bus load forecasting more, while each algorithm has its own field of application [4]. However, these artificial intelligence algorithms are not only relatively complicated as well as fluctuated in forecasting accuracy but also exist some limitations in practical application [5]. In view of this, this paper first based on typical area daily load curve, classifies regional bus load with subtractive clustering, and builds bus load matching model for clustering load; On this basis, with the help of LSSVM which has better forecasting performance for small samples, bus load forecasting model has been built; Finally, it verifies the scientific nature and effectiveness of load matching and forecasting model constructed herein by example.

\section{Bus load matching model for clustering load}

This section combines a number of bus loads meeting certain conditions into a clustering load. First it predicts clustering load, then it assigns predictive value to each bus based on proper matching model. This method on the one hand, could divide a large number of bus loads into several less classes, reducing the number of forecasting targets and reducing calculation amount; On the other hand, due to 
the load base of clustering load is higher as well as load structure is more stable, the fluctuation of internal nodes load could be cancelled each other to improve their regularity, therefore it is more predictable and helpful to improve forecasting accuracy.

Based on daily load curve, it extracts characteristic quantities including daily maximum load, daily minimum load, daily average load, and daily load ratio to conduct fuzzy clustering. This paper adopts subtractive clustering which can find the initial cluster center according to effects of each data point in each dimension on the cluster center, as well as automatically produce better number of clusters. It not only avoids falling into local optimal solution, improve the speed of clustering, but also does not need to pre-determine the number of clusters. Specific clustering process is as follows:

(1) Calculate the density indexes for each data point [6]:

$$
M_{i}=\sum_{j}^{n} \exp \left[-\frac{\left\|a_{i}-a_{j}\right\|^{2}}{\left(0.5 d_{a}\right)^{2}}\right]
$$

Where $d_{a}$ is the neighborhood radius of this point. Select the highest density index data point $a_{c 1}$ as the first cluster center, around which there are a plurality of adjacent sample points.

(2) Correct density indexes of the remaining sample point according to the cluster center selected in -th:

$$
M_{i}=M_{i}-M_{c k} \exp \left[-\frac{\left\|a_{j}-a_{c k}\right\|^{2}}{\left(0.5 d_{b}\right)^{2}}\right]
$$

Where $d_{b}$ defines a significantly reduced neighborhood of density index function, usually taken. According to the adjusted results, select the highest density index data point as a new cluster center.

(3) Judge if the exit condition is satisfied:

$$
\frac{M_{c k+1}}{M_{c 1}}<\partial
$$

If it is not satisfied, go to step (2). Where $\partial$ determines the final number generated of initialization clustering centers.

In fact, bus load forecasting for clustering load constitutes a predictive model similar to the one based on system load distribution, which is namely assign predictive value of clustering load to the internal each bus. Since the clustering load is very similar to the internal load curves of each bus, it is possible to use the relatively simple matching model, which is estimated by smoothing recent matching factors.

The child bus $i$ belongs to the clustering load $f$, then the bus $i$ at time $t$, predictive matching factor $\hat{\lambda}_{l, t}{ }^{f}$ and the actual matching factor $\lambda_{l, t}{ }^{f}$ satisfy the following formula:

$$
\hat{\lambda}_{i, t}{ }^{f}=\lambda_{\mathrm{i}, t}{ }^{f}\left(1+\ell_{i, t}{ }^{f}\right)
$$

Where, $\ell_{l, t}{ }^{f}$ is the predictive error coefficient, which is inversely proportional to the regularity of actual matching factor, and the actual matching factor [7] is calculated as follows:

$$
\lambda_{\mathrm{i}, t}{ }^{f}=\frac{1}{1+\sum_{j \neq i} \frac{b_{j, t}{ }^{f}}{b_{i, t}{ }^{f}}}
$$

Where $b_{i, t}{ }^{f}$ is the actual load value of the bus $i$ at time $t$.

Further, obtain the predictive values $\hat{b}_{i, t}{ }^{f}$ of bus $i$ at time $t$ with the error coefficients' calculation formula, shown as follows:

$$
\hat{b}_{i, t}{ }^{f}=\hat{\lambda}_{i, t}{ }^{f} \hat{b}_{t}^{f}
$$

Where, $\hat{b}_{t}^{f}$ is the predictive value of clustering load $f$ at time $t$. 


\section{Short-term bus load forecasting model based on LSSVM}

LSSVM is an extension of support vector machine (SVM), which project low-dimensional nonlinear input vector to a high-dimensional space to construct optimal decision surface. Then it applies structural risk minimization principle, changing SVM inequality operation into equation solving operation, which reduces the computational complexity and speed up the processing speed.

Steps of short-term bus load forecasting model based on LSSVM are as follows:

(1) Structure of model training sample set

Collect bus daily load data and select load value of forecasting day-ahead observation time point, select load value of forecasting day-ahead observation previous time point and so on as samples.

(2) Data normalization

To improve training speed and forecasting accuracy, normalize the input and output data of the sample set, shown as formula (7).

$$
\bar{x}_{i}=\frac{x_{i}-x_{\min }}{x_{\max }-x_{\min }}
$$

(3) Choice of kernel function

$$
K\left(\mathrm{x}_{i}, \mathrm{x}_{j}\right)=\exp \left(-\frac{1}{2 \sigma^{2}}\left\|\boldsymbol{x}-\boldsymbol{x}_{i}\right\|^{2}\right)
$$

(4) Training and simulation. Train and study historical data after normalization with the aforesaid bus load classification data based on LSSVM intelligent forecasting model, and obtain the predictive value and the actual value, then calculate the relative error according to formula (9).

$\ell=|(\hat{Y}-Y) / Y| \times 100 \%$

Where, $Y$ represents the actual value, and $\hat{Y}$ represents the predictive value.

(5) Forecasting results analysis

\section{Case study}

Conduct cluster analysis with actual load data of a certain region in Beijing, Tianjin and Hebei province, and the grid bus load of this region is divided into following three categories:

(1)The first category is the bus daily load curve with obvious load peaks and valleys, which is relatively smooth, fluctuations changing obviously. This type of load mostly belongs to residential load and institution load, including minority businesses, shopping centers and hotels and other types of loads.

(2)The second category is the gentle bus daily load curve and load level is always in a higher position, regardless of time variations in a day. This type of load mostly belongs to factories and enterprises with continuous or three-shift production.

(3)The third category is the bus daily load curve with large data mutation, poor stability, and no obvious periodicity distribution, which usually belongs to the loads affected by a number of random factors, such as impact load or large user-specific load.

On this basis, load forecasting with the LSSVE model are shown in Figure 1, Figure 2 and Figure 3.

Combined with these three figures, it can be seen that forecasting errors between the first two categories' predictive values and the load actual value are relatively small with better forecasting. The error between bus load forecasting results of the third category and the actual value is relatively large, with poor forecasting.

On this basis, conduct forecasting for the regional total daily load curve respectively by means of two methods. Method One: based on the clustering results, respectively conduct forecasting for each type of load (which is total bus loads in the category), and then add up forecasting results of three categories; Method two: conduct forecasting directly for total load in the area. The forecasting results are shown in Figure 4. 


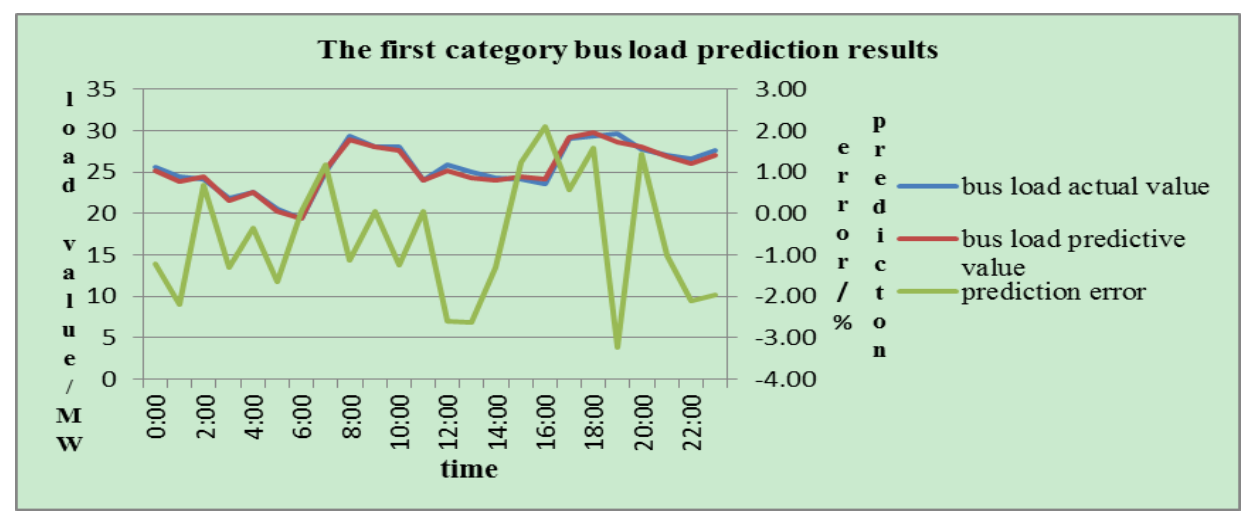

Fig.1 The first category bus load forecasting results.

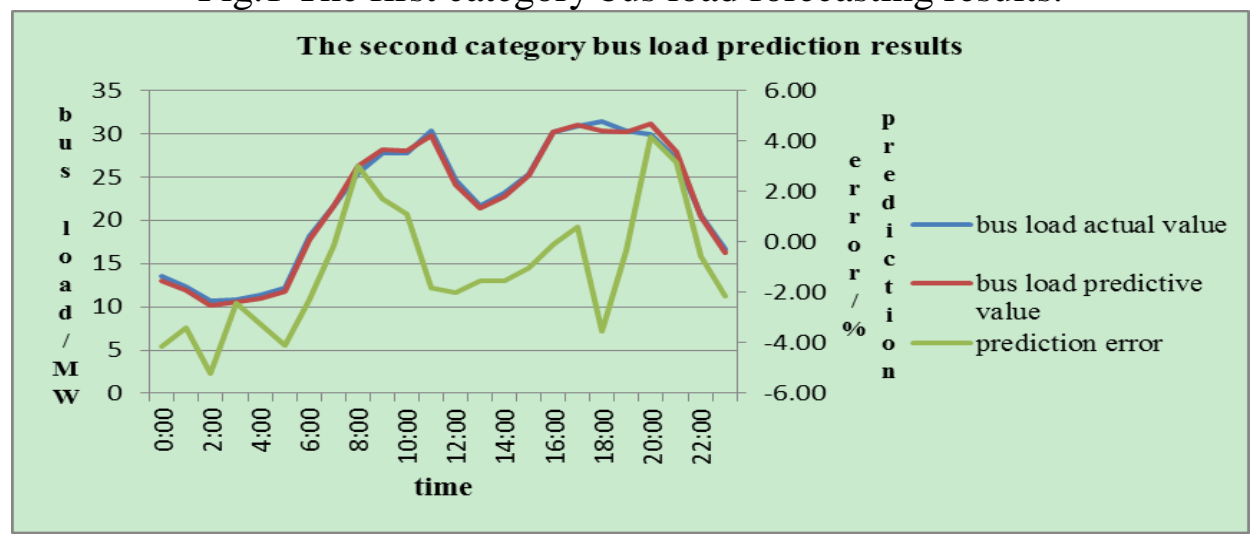

Fig. 2 The second category bus load forecasting results.

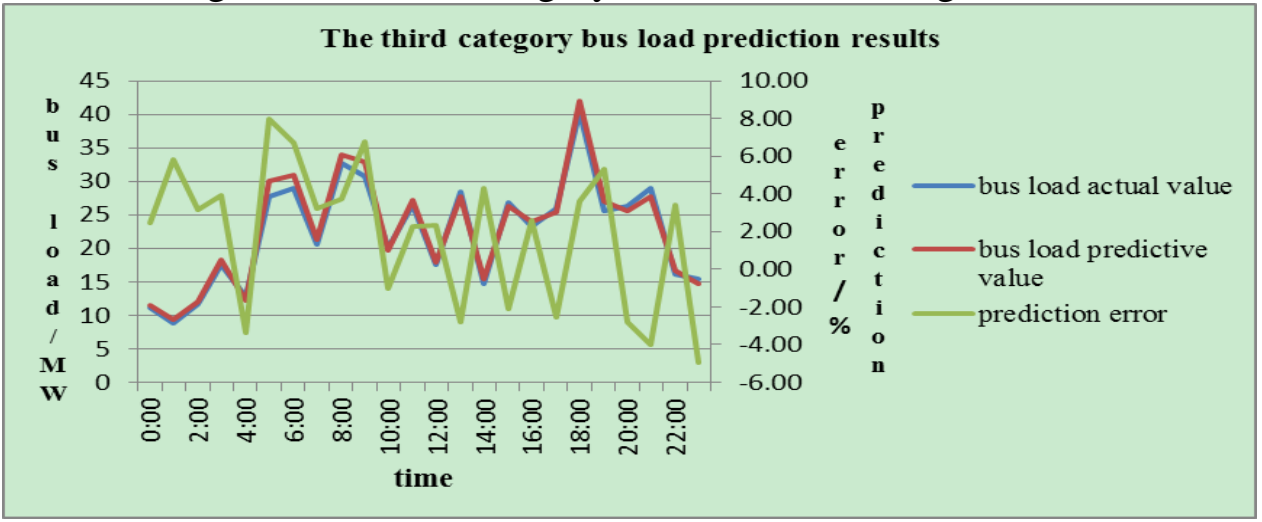

Fig.3 The third category bus load forecasting results.

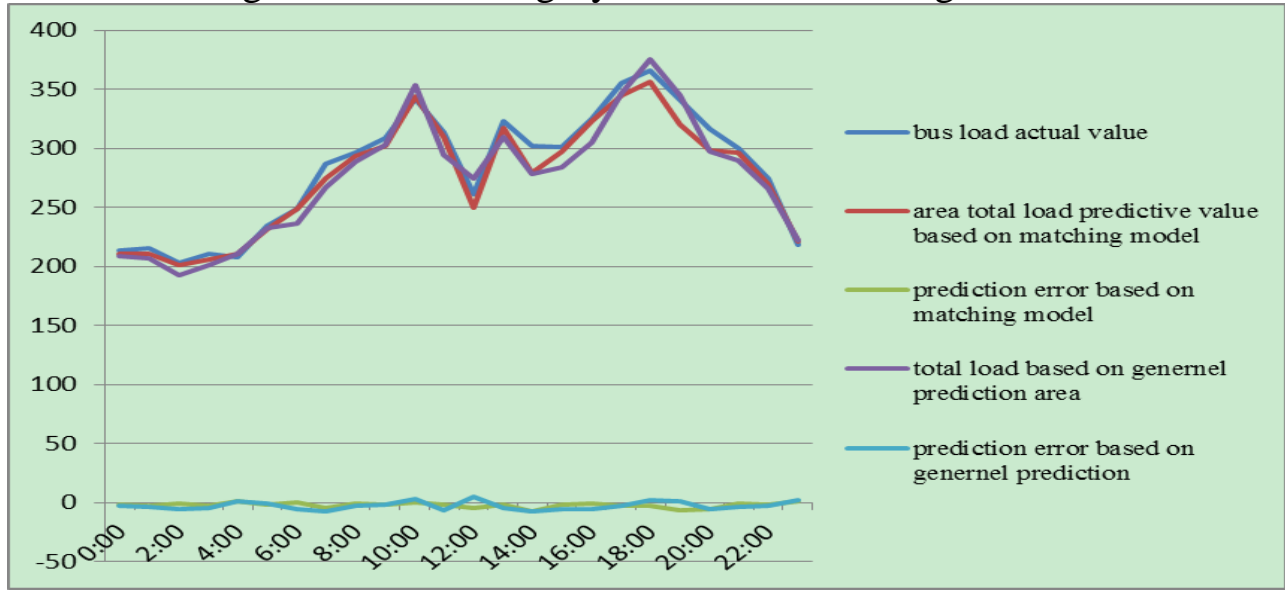

Fig.4 Total regional load forecasting results

As can be seen from the figure, compared with the method directly conducting forecasting for the regional total load, adapting the first method based on the clustering results, which respectively conducts load forecasting for each category (namely the total bus load of that category) and adds up the load forecasting results of three categories aggregation method for predicting the results obtained 
after three load is closer to the actual value and the forecasting error is smaller as well as precision is higher.

\section{Conclusion}

Short-term bus load forecasting is an important foundation to achieve lean scheduling for the power system, helping power grid to develop energy-saving plan. In addition, bus load forecasting research helps the system to control the operation and management for the current decentralized load management. Firstly, this paper builds bus load matching model for clustering load; On this basis, it builds bus load forecasting model with LSSVM; Finally, it verifies the scientific nature and effectiveness of the load forecasting model built herein by example, and the predictive results shows that the method of classifying each bus load based on its characteristics scientifically, predicting and then re-synthesizing the clustering load can be used as a method of improving system load forecasting model, which promotes the system load forecasting results more scientific and accurate.

Factors of regional grid bus load forecasting are complicated. How to combine the actual situation, and consider the impacts of plan maintenance, meteorological information, economic structure and other factors is worthy of further study.

\section{Acknowledgements}

This paper was supported by Science and Technology Project of State Grid Corporation of China (Research on the effects of air pollution prevention and control on the power supply and demand of Beijing-Tianjin-Hebei Region).

\section{References}

[1]. Xueqian Fu, Haoyong Chen, Guote Liu, Xiaoming Jin. Power Quality Comprehensive Evaluation Method for Distributed Generation [J].Proceedings of the CSEE, 2014, 25(3): 4270-4276.

[2]. Limei Zhang, Wei Tang, Shaolin Wang. Distributed Generators Planning Considering Benefits for Distribution Power Company and Independent Power Suppliers[J].Automations of electric power system, 2011, 18(04): 23-28.

[3]. Bozhong Wang. Research and Application of Ultra-Short Term Bus Load Forecasting [D]. North China Electric Power University, 2012.

[4]. Kassaei H.R, Keyhani A, Woung T, et al.A Hybrid Fuzzy, Neural Network Bus Load Modeling and Predication.IEEE Transactions on Power Systems, 1999, 14(2): 718-724.

[5]. Huilan Jiang, Xiaojin Liu, Ying Guan. Short-Term Load Forecasting Based on Hard-C Clustering Algorithm and Support Vector Machine[J].Power System Technology, 2006,30(8):81-85.

[6]. Feng Liao, Qingliang Liu, Hui He, Yiming Cheng, Congying Xu, Jiangang Yao. Bus Load Forecasting Based on Improved Grey Model and Meteorological Elements [J]. Power System Technology, 2011, 10:183-188.

[7]. Qian Sun. Research on Forecasting Technology for Bus Load and Impacts of Its Characteristics on Power Grids [D]. Hunan University, 2013. 